\section{Available online at www.sciencedirect.com $\because$ ScienceDirect Journal of Hydrodynamics}

2010, 22(5), supplement :391-397 DOI: $10.1016 / \mathrm{S} 1001-6058(09) 60225-0$

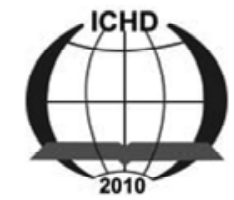

www.sciencedirect.com/ science/journal/10016058

\title{
A study on separation performance of a vortex finder in a liquid-liquid cylindrical cyclone
}

\author{
Shi-ying Shi, Ying-xiang Wu, Jun Zhang, Jun Guo, Shu-jing Wang \\ Institute of Mechanics, Chinese Academy of Sciences, Beijing, China \\ *E-mail: shishiying123@163.com
}

Error! Reference source not found.ABSTRACT: This paper outlines the influence of design parameters such as different diameter, different shapes and insertion depth of a vortex finder on Liquid-Liquid Cylindrical Cyclone (LLCC) oil-water separation characteristics through experiments and numerical simulations. Through numerical simulations, the separation efficiency for water-dominated flow conditions is obtained and the results show that there exists an optimal shape, diameter and insertion depth of a vortex finder in cylindrical cyclones under certain conditions. In our laboratory, a $100 \mathrm{~mm}$ inner diameter LLCC is adopted to test the influence of different insertion depths of the vortex finder and the results again indicate that an optimal insertion depth exists. The numerical results agree well with the experimental data of Rajkumar S et al. . So numerical simulations can be used to predict the complex flow behavior, the separation efficiency in a LLCC for optimizing the structure and provide some design guidance for their industrial application as the oil-water separators on offshore platforms and oil field inland.

KEY WORDS: Liquid- Liquid Cylindrical Cyclone; vortex finder strucure; oil-water separation; experiment; numerical simulation.

\section{INTRODUCTION}

Hydrocyclones are widely used in many areas such as mineral, chemical, textile, pharmaceutical and petrochemical industries. Their specific features like low weight, low cost,no moving parts, easy operation, and high separation efficiency make them popular for oil-water separation in the offshore fields.

The Liquid-Liquid Cylindrical Cyclone is a new technology for oil-water separation. It is a section of vertical pipe with a horizontal inlet through which the two-phase oil-water mixture enters. The swirling motion in the LLCC produces a centrifugal separation,whereby, an oil-rich stream exits through the top(overflow) and a water-rich stream leaves the system through the bottom (underflow) ${ }^{[1]}$. A schematic of a LLCC is given in Figure 2.
Several experimental and computational investigations have been conducted to determine the effects of design parameters on the cyclone performance. Listewnik ${ }^{[2]}$ got the oil-water separation efficiency in a cylindrical hydrocyclone with four inlets. Seyda ${ }^{[3]}$ simulated oil-water separation in a small cylindrical tube. $\mathrm{Chu}^{[4]}$ considered the effect of different vortex finder designs for a hydrocyclone on separation efficiency. A Belaidi ${ }^{[5]}$ revealed the effects of the mean feed droplet size on a de-watering hydrocyclone with a vortex finder. Rajkumar S.Mathiravedu ${ }^{[6]}$ presented that clear water with a higher split ratio was produced by using a vortex finder. Minghu Jiang ${ }^{[7]}$ studied hydrocyclones with vortex finders and found that different geometric parameters of hydrocyclones should be selected for different types of operating conditions for a better separation effect. Lucia Fernandez Martinez ${ }^{[8]}$ reported that an optimum vortex finder length existed for hydrocyclone solid-liquid separation.

The above literature reflects that the influence of vortex finder designs, optimum insertion depth and diameter of the LLCC has not yet been systematically studied before. In the present work, the influence of the vortex finder diameter vinsertion depth and different vortex finder designs under various mean oil droplet diameters v velocity and oil concentration at the inlet in the LLCC was studied systematically using CFD techniques and a number of experiments were conducted.

\section{MATHEMATICAL MODEL}

Despite the simplicity of the hydrocyclone in terms of construction, the internal features of the vortex breakdown phenomenon and reverse flow make its fluid-dynamic behaviour rather complex ${ }^{[9]}$. Thus the choice of turbulence models to predict the above flow 
behaviour remains a disputed issue. Hirai et al. ${ }^{[10]}$ observed that the results of the modified $\kappa-\varepsilon$ model gave a better performance than the $\kappa-\varepsilon$ model and the mentioned results were comparable with the RSM results. A.Malhotra ${ }^{[11]}$ showed that employing the conventional $\kappa-\varepsilon$ model to predict the flow field in a hydrocyclone proved to be inadequate. P.He et.al. ${ }^{[12]}$ showed that the modified $\kappa-\varepsilon$ model produced good agreement of the hydrocyclone's separation efficiency with experimental data. Grady et al. ${ }^{[13]}$ made use of the Reynolds stress turbulence closure model and the algebraic slip multiphase model to predict the velocity field and separation efficiency of a $10 \mathrm{~mm}$ deoiling hydrocyclone. C.Oropeza-Vazquez et al. ${ }^{[1]}$ developed a new mechanistic model to calculate the separation efficiency in a LLCC. Andrew Escue et al. ${ }^{[14]}$ found that the RNG $\kappa-\varepsilon$ model was in better agreement with experimental velocity profiles for low swirl.

The difference between the RNG $\kappa-\varepsilon$ model and RSM is their methods of calculating the Reynolds stresses in the RANS equations. The advantage of using the RNG $\kappa-\varepsilon$ model lies in its simplicity of implementation. Yet, it has been proved to be able to capture the physics of the flow correctly ${ }^{[12]}$. In the RSM, the Reynolds stresses are solved directly through six partial differential equations, thus it needs a lot of computer memory and time to achieve convergence. To save time the RNG $\kappa-\varepsilon$ model is chosen in this paper.

In the Eulerian-Eulerian mutiphase model, each phase is treated as a continuum and characterized by averaged conservation equations. The mixture model makes use of an algebraic slip formulation. It is a simplification of the Eulerian approach used for multiphase flow and allows consideration of the problem at low computational cost. Owing to this fact, the liquid-liquid two-phase flow is described by using the Mixture approach.

All the equations are constructed under the following assumptions: droplets are spherical and have the same diameter; coalescence and fragmentation of droplets do not happen; the flow field is isothermal and steady; the fluid is incompressible.

According to the mass conservation law, the continuity equation is as follows:

$$
\begin{aligned}
& \nabla \cdot\left(\rho_{m} \overrightarrow{v_{m}}\right)=0 \\
& \rho_{m}=\alpha_{o} \rho_{o}+\alpha_{w} \rho_{w} \\
& \overrightarrow{v_{m}}=\frac{\alpha_{o} \rho_{o} \overrightarrow{v_{o}}+\alpha_{w} \rho_{w} \overrightarrow{v_{w}}}{\rho_{m}}
\end{aligned}
$$

$$
\begin{aligned}
& \nabla \cdot\left(\rho_{m} \vec{v}_{m} \overrightarrow{v_{m}}\right)=-\nabla p+\nabla \cdot\left[\mu_{m}\left(\nabla \overrightarrow{v_{m}}+\nabla \overrightarrow{v_{m}^{T}}\right)\right]+ \\
& \rho_{m} \vec{g}+\nabla \cdot\left(\sum_{k=1}^{2} \alpha_{k} \rho_{k} \vec{v}_{d r, k} v_{d r, k}\right) \\
& \mu_{m}=\alpha_{o} \mu_{o}+\alpha_{w} \mu_{w} \\
& \vec{v}_{d r, o}=\vec{v}_{w o}-\vec{v}_{m} \\
& \overrightarrow{v_{w o}} \text { is obtained by algebraic slip formula: } \\
& \vec{v}_{w o}=\tau_{w o} \vec{\alpha}
\end{aligned}
$$

According to Manninen theory:

$$
\begin{aligned}
& \vec{\alpha}=\vec{g}-\left(\vec{v}_{m} \cdot \nabla \vec{v}_{m}\right) \\
& \tau_{w o}=\frac{\left(\rho_{m}-\rho_{o}\right) d_{o}^{2}}{18 \mu_{o} f_{d r a g}}
\end{aligned}
$$

Drag force function is defined by the best available model-Morsi and Alexander:

$$
\begin{aligned}
& f_{\text {drag }}=\frac{C_{D} \mathrm{Re}}{24} \\
& C_{D}=a_{1}+\frac{a_{2}}{\operatorname{Re}}+\frac{a_{3}}{\operatorname{Re}^{2}} \\
& \operatorname{Re}=\frac{\rho_{o}\left|\overrightarrow{\mathbf{v}}_{w}-\overrightarrow{\mathbf{v}}_{o}\right| d_{o}}{\mu_{o}}
\end{aligned}
$$

$\kappa 、 \mathcal{E}$ equations are as follows:

$$
\begin{aligned}
& \nabla \cdot\left(\rho_{m} \vec{v}_{m} k\right)=\nabla \cdot\left(\frac{\mu_{t, m}}{\sigma_{k}} \nabla k\right)-\rho_{m} \varepsilon+G_{k, m} \\
& \nabla \cdot\left(\rho_{m} \vec{v}_{m} \varepsilon\right)=\nabla \cdot\left(\frac{\mu_{t, m}}{\sigma_{\varepsilon}} \nabla \varepsilon\right)+ \\
& \frac{\varepsilon}{k}\left(C_{1 \varepsilon} G_{k, m}-C_{2 \varepsilon} \rho_{m} \varepsilon\right) \\
& G_{k, m}=\mu_{t, m}\left[\nabla \vec{v}_{m}+\left(\nabla \vec{v}_{m}^{T}\right)\right] \nabla \vec{v}_{m} \\
& \mu_{t, m}=\rho_{m} C_{\mu} \frac{k^{2}}{\varepsilon}
\end{aligned}
$$

where the five constants in the equations are:

$$
\begin{aligned}
C_{1 \varepsilon} & =1.44, \quad C_{\mu}=0.0845, \quad C_{2 \varepsilon}=1.92, \\
\sigma_{k} & =1.0, \quad \sigma_{\varepsilon}=1.3
\end{aligned}
$$

The volume fraction equation is given by:

The momentum equations may be simplified as: 


$$
\nabla \cdot\left(\alpha_{o} \rho_{o} \vec{v}_{m}\right)=-\nabla \cdot\left(\alpha_{o} \rho_{o} \vec{v}_{d r, o}\right)
$$

Since the governing equations are elliptic, it is necessary to define boundary conditions for all the boundaries of the flow conditions: (1)uniform velocity boundary conditions are used at the inlet, and the velocity is perpendicular to the inlet cross-section. The turbulence intensity I, is defined as:

$$
\begin{aligned}
& I=\frac{v_{m} L \mu_{m}}{\rho_{m}} \\
& L=\frac{4 A}{S}
\end{aligned}
$$

(2) The outlet boundary conditions are assumed to be outflow, that implies zero gradient for any flow variable in the direction parallel to the outlet pipes; (3) The wall of the LLCC is treated as an impermeable and no-slip surface so that the velocity and the turbulence kinetic energy are zero.

Based on the finite-volume method, equations are converted to algebraic equations with an upwind difference scheme. The flow field inside the LLCC is obtained by the SIMPLE algorithm. The convergence criteria are not reached until the volumetric ratio of the oil phase at the two outlets remains still and the simulations are carried out for about 20,000 iterations. To verify the above model, the oil/water separation in the LLCC is numerically simulated, and the results are compared with the experimental data obtained by Rajkumar $\mathrm{S}^{[6]}$. Fig 1.(a) is their experimental equipment. During their experiment, the temperature is $26.7^{\circ} \mathrm{C}, \rho_{o}=855.56 \mathrm{~kg} / \mathrm{m}^{3}, \mu_{o}=0.01576 \mathrm{~kg} / \mathrm{m} \cdot \mathrm{s}$, $\rho_{w}=998.0 \mathrm{~kg} / \mathrm{m}^{3}, \mu_{w}=0.001 \mathrm{~kg} / \mathrm{m} \cdot \mathrm{s}$, the superficial water velocity is maintained at $0.4 \mathrm{~m} / \mathrm{s}$ and the superficial oil velocity is maintained at $0.08 \mathrm{~m} / \mathrm{s}$ (oil concentration at inlet $=16.67 \%$ ). Fig. 1 (b) compares the simulation result of oil-water distribution and the phenomenon observed from the experiment. Fig.1 (c) compares the predicted and the experimental data obtained by Rajkumar Set al.. When the flow rate of the overflow changes, the error between the numerical results and the experimental data is within $10 \%$, the agreement between the simulation and the experiment is satisfactory. So the above model can be considered valid to study the flow in the LLCC.

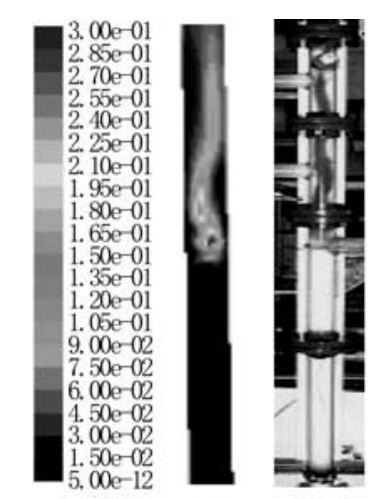

(a)

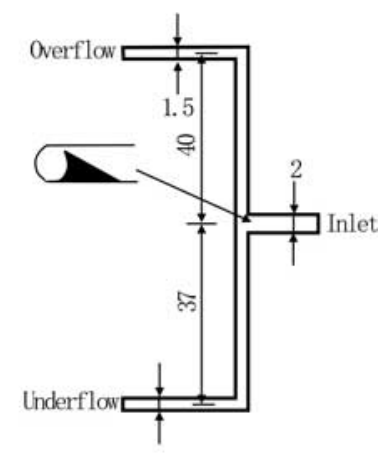

(b)

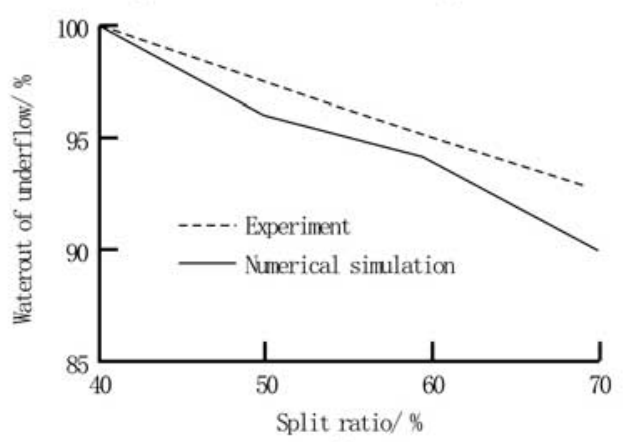

(c)

Fig. 1 Comparisons between numerical simulation and experiments

\section{GEOMETRIC MODEL}

The design structure of the prototype for the numerical simulation is shown in Fig. 2 (unit: $\mathrm{mm}$ ). As can be seen, the overflow tube is $500 \mathrm{~mm}$ in length. The five different diameters of the vortex finder are defined in Table. 1. Four shapes of the vortex finders are illustrated in Fig. $3^{[4]}$.

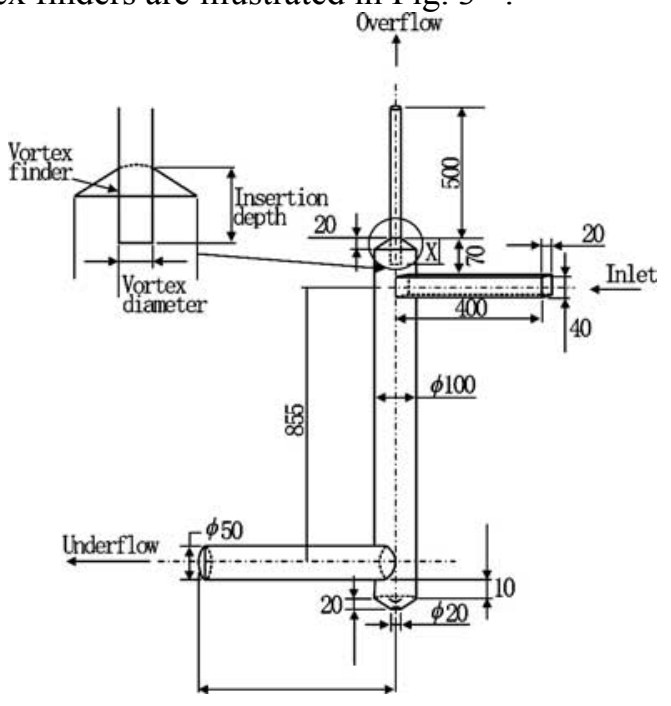

Fig. 2 LLCC design 


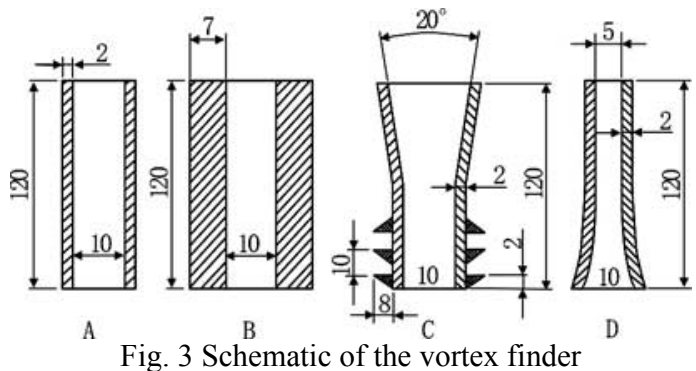

The following designs of vortex finder are based on type A. All the other parameters are the same except for the diameter.

Table 1.Vortex finder diameter design table

\begin{tabular}{|c|c|c|c|c|c|}
\hline $\begin{array}{c}\text { The diameter } \\
\text { of vortex finder } \\
\mathrm{d} / \mathrm{mm}\end{array}$ & 20 & 30 & 40 & 50 & 60 \\
\hline label & A1 & A2 & A3 & A4 & A5 \\
\hline
\end{tabular}

\section{RESULTS AND DISCUSSION}

There exist three groups of variables that affect the oil/water separation in the LLCC: dimensional variables; such as the length, diameter and shape of the vortex finder, etc.,operational variables; the mixture velocity entering the LLCC, etc., feed stream variables; for example the oil viscosity, oil concentration, droplet mean diameter and so on.

Before considering the performance of the LLCC with respect to the various variables, let us define some criteria to evaluate the LLCC.

1) separation efficiency $E$ :

$E=\frac{k_{o}}{k_{i}}$

It can be seen that as $k_{o}$ increases, E increases. The separation efficiency is a measure of the LLCC for a particular oil and water flow rate with a given oil droplet size at the inlet.

2) Split ratio $F$ :

$F=\frac{Q_{o}}{Q_{i}}$

3) pressure drop $\Delta P$ :

$\Delta P=p_{i}-p_{u}$

\subsection{Experimental system}

An experimental system was established at the Laboratory of Applied Fluid Dynamics in Chinese Academy Sciences Fig 4.(a). Fig 4.(b) shows the experimental system where the oil-water separation inside LLCC were conducted. The LLCC (Fig.4 (c)) was made of plexiglass to enable visual observation of oil-water separation. The LLCC was a $1600 \mathrm{~mm}$ long, $100 \mathrm{~mm}$ ID vertical pipe, with $100 \mathrm{~mm}$ ID horizontal inlet. The inlet was attached to the LLCC $210 \mathrm{~mm}$ below its top. A nozzle was located at the LLCC inlet, causing the flow to enter the LLCC tangentially. The inlet slot area was $10 \%$ of the inlet full bore cross sectional area. A $50 \mathrm{~mm}$ ID concentric pipe located at the top was used as the overflow. A $15 \mathrm{~mm}$ ID concentric pipe with the wall thickness of $5 \mathrm{~mm}$ located at the top was used as the vortex finder and the $65 \mathrm{~mm}$ ID pipe located at the bottom as the underflow. Valves in the overflow allowed the control of the split-ratio F. The water and oil flow rate were measured in the test utilizing an electromagnetic flow meter and a roots flowmeter respectively. Oil is mixed with with water in a horizontal Y-junction after they flow out from their tank, and then they enter the LLCC through a reducing area nozzle, increasing their velocity to form a swirling motion in the LLCC. Due to a centrifugal separation, an oil-rich stream exits through the overflow and a water-rich stream leaves through the underflow.

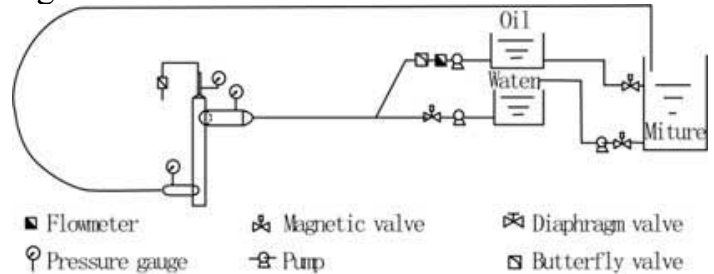

(a)
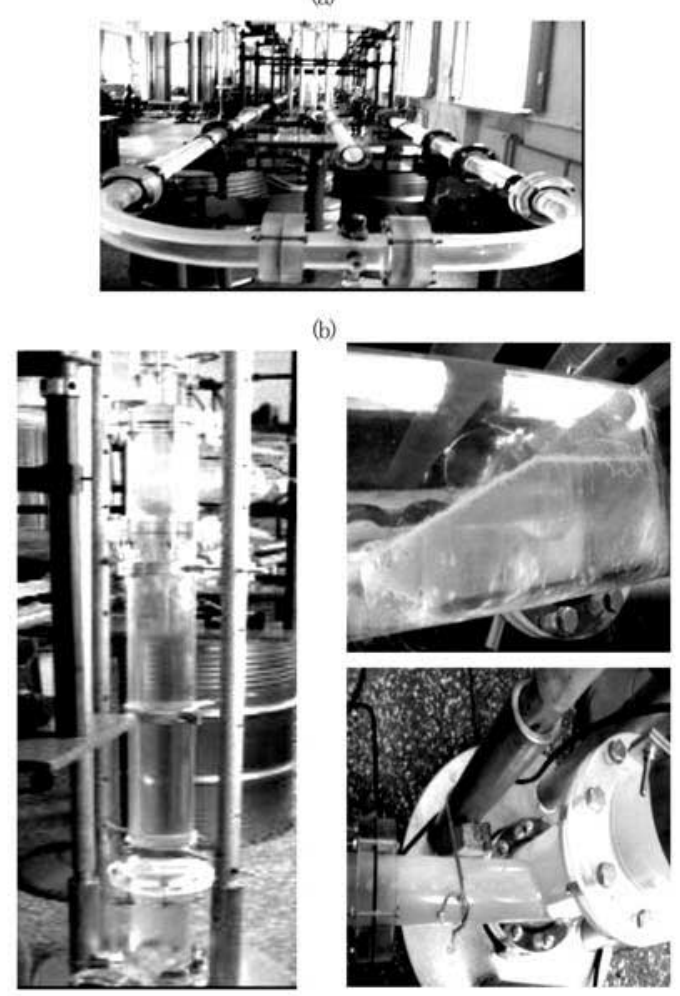

(c)

Fig.4 Experimental system for oil-water separation in the LLCC 
When the superficial water velocity is $0.71 \mathrm{~m} / \mathrm{s}$ and the superficial oil velocity is $0.07 \mathrm{~m} / \mathrm{s}$ (oil content at the inlet $=9.3 \%$ ), the oil droplets move to the top of the pipe and the water in a continuous phase. An oil in water dispersion flows through the top of the pipe and a free-water layer flows through the bottom. Keeping $F=0.2$, Fig. 5 shows the effect of five insertion lengths $\mathrm{x}: 0 \mathrm{~mm}, 140 \mathrm{~mm}, 260 \mathrm{~mm}, 376 \mathrm{~mm}, 519 \mathrm{~mm}$ on the oil volume fraction at the overflow. The oil volume content when vortex is adopted is higher than the LLCC without vortex finder. Maybe by introducing the vortex finder, some water near the wall exit directly through the overflow forming a shortcircuited flow at the top wall of the LLCC. An insertion depth of $376 \mathrm{~mm}$ which lies about $20 \mathrm{~mm}$ below the inlet performs better than other depths. This conclusion is the same as the numerical simulation result in our earlier paper ${ }^{[15]}$. When the insertion length is too long, some oil from the core is taken away by the water while waiting to exit through the overflow; when the insertion length is too short, some water from the inlet exit directly through the vortex finder and decreases the oil volume fraction in the overflow.

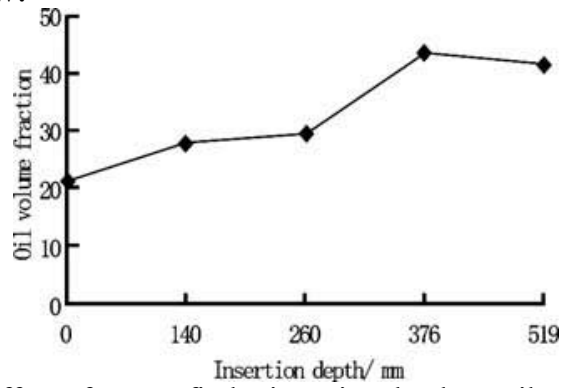

Fig. 5 Effect of vortex finder insertion depth on oil separation

\subsection{Influence of vortex diameter}

Fig.6 and Fig.7 show the effect of vortex finder diameter on the oil-water separation. Under the same operational conditions, the oil volume fraction increases and then decreases as the diameter becomes larger. When vortex diameter is $20 \mathrm{~mm}$, the separation efficiency of LLCC is highest. For a given oil content at the inlet with a small vortex diameter, some oil is taken away by water into the underflow while waiting to exit from the overflow and such movement has a negative impact on the oil core. When the vortex diameter is too large and the oil content at the inlet is fixed, the water outside the oil core flows directly into the vortex finder, thus the oil content at the overflow decreases.
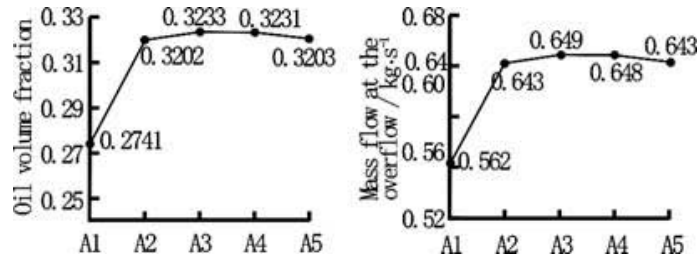

Fig. 6 Effect of vortex finder diameter on separation
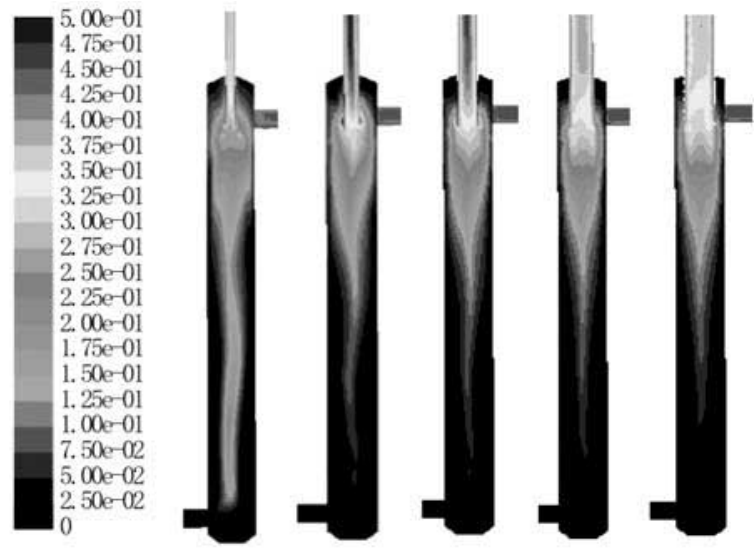

Fig. 7 Distribution of oil volume fraction

A5

\subsection{Influence of vortex shapes on pressure drop}

When the insertion length and the diameter of vortex finder are $120 \mathrm{~mm}$ and $20 \mathrm{~mm}$ respectively, Fig 8 . shows the effect of vortex shapes on the pressure drop, Shape D produces the largest pressure drop, B has the same effects as C. In addition, the smallest pressure drop that could be achieved out of all considered shapes is that with shape A. Figure 9. Shows the effect of vortex shapes on oil-water separation. As can be seen, the underflow of $\mathrm{A}$ has the least and $\mathrm{D}$ has the most oil. This may be the result of a frictional pressure drop caused by the the various shapes of vortex finder.

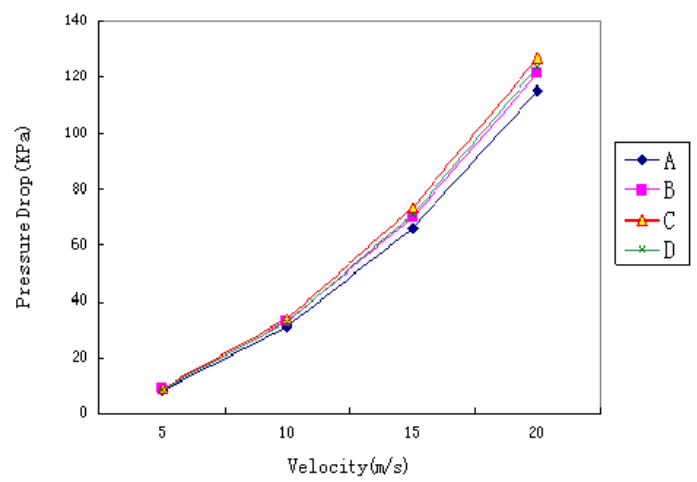

Fig. 8 Effect of vortex finder shapes on pressure drop

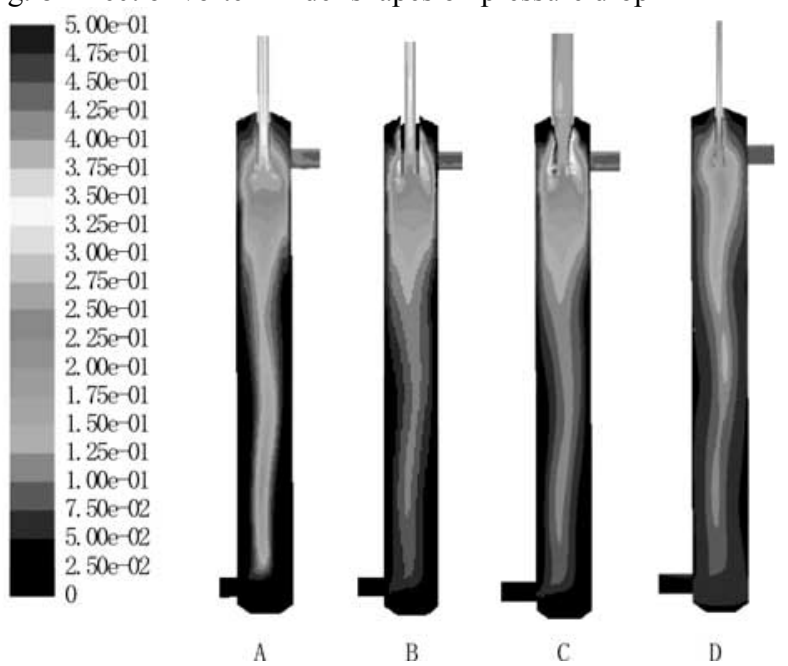

Fig. 9 Distribution of oil volume fraction 


\subsection{Influence of velocity on separation}

Fig.10 shows that separation efficiency increases when the inlet phase speed becomes faster. Besides A has the best phase separation capacity among these designs, while D improves the LLCC performance the least. It is not hard to understand that when the phase velocity increases, the centrifugal forces on the oil droplets increase and move the droplets toward the center of the LLCC and drives them to exit through the overflow.

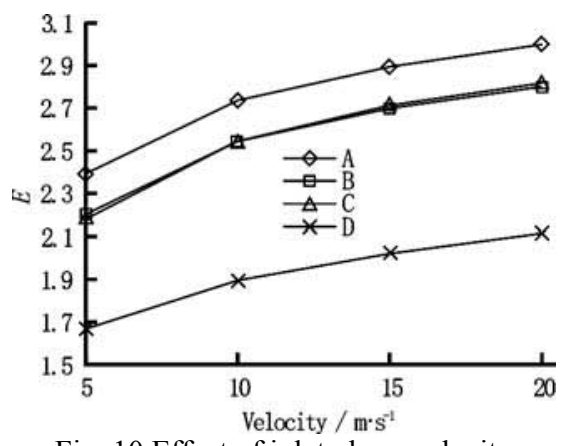

Fig. 10 Effect of inlet phase velocity

\subsection{Influence of oil droplet size}

The effect of changing the mean droplet diameter when the mixture velocity is $15 \mathrm{~m} / \mathrm{s}$, the oil phase volume fraction is $10 \%$, the effect of the mean oil droplet size on the separation efficiency using different vortex finder shapes has been presented in Fig.11. It is observed that the droplet size affects the LLCC oil/water separation significantly. That means that to improve LLCC separation efficiency, increasing the mean droplet diameter or trying to avoid droplet break into very small ones is a practical method. Another conclusion is that when the droplet size decreases, the gap between different designs of vortex finder designs narrows. In other words, when the oil droplet size is small, the designs of the vortex finder does not make much difference. When the oil droplet size is large, A performs better than B,C or D. $\mathrm{B}$ and $\mathrm{C}$ have almost the same effect.

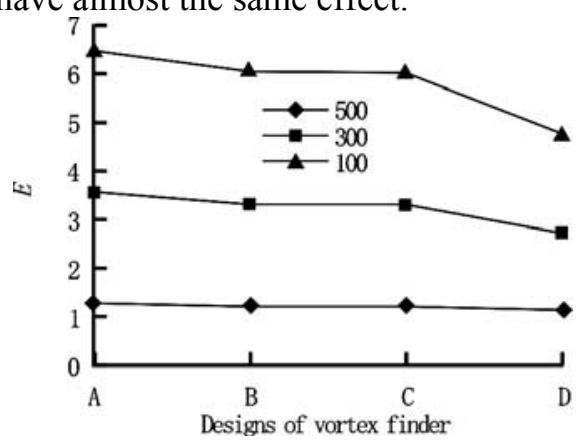

Fig. 11 Influence of mean oil droplet size on efficiency

\subsection{Influence of oil concentration}

The effects of oil concentration on the separation efficiency using different vortex finder designs when the other conditions are kept the same, are shown in Fig.12. When the oil concentration at the inlet increases, efficiency decreases. Having a constant droplet diameter and increasing the oil concentration means an increase in the droplet number. As interaction between droplets increases, the competition for moving toward the core and exit via the overflow tube becomes more fierce. This is why the efficiency is reduced

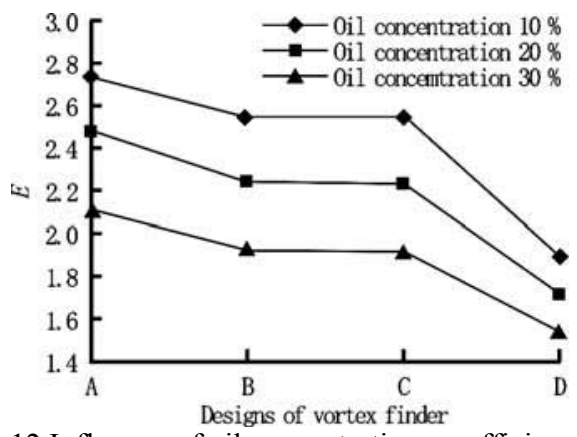

Fig. 12 Influence of oil concentration on efficiency

\section{CONCLUSIONS}

The influence of a vortex finder's length, diameter and designs on the LLCC is studied. Two-phase liquid-liquid flow through a LLCC is simulated with the Mixture multiphase model, and the RNG $\kappa-\varepsilon$ model for steady flows is computed. The simulations reach the following conclusions:

Improving the efficiency of a LLCC through changing the length of the vortex finder is feasible. The optimum length of vortex finder was found to be situated about twenty millimeters under the lowest point of the feed inlet section.

When increasing the diameter of the vortex finder, the overflow oil content and oil mass flow rate increase at first and then decrease. A forty millimeters vortex diameter offers the best performance.

Increasing the inlet phase velocity could raise efficiency, between different shapes of vortex finder, shape A has the smallest pressure drop with the best separation efficiency, while D has the opposite effect. Shapes B and C have a performance similar to each other and an efficiency between that of shapes A and D.

Oil droplet size has a significant impact on separation. Increasing the mean droplet diameter or trying to avoid droplet break into very small ones is a practical method to improve LLCC separation efficiency.

When the oil concentration increases from $10 \%$ to $30 \%$, the LLCC separation efficiency decreases.

\section{NOMENCLATURE}

$$
\rho=\text { density }
$$


$\rightarrow$

$\vec{v}=$ average speed

$\mu=$ dynamic viscosity

$\rightarrow$

$v_{d r, o}=$ drift speed of oil phase

$\overrightarrow{v_{w o}}=$ relative speed between oil and water

$\rightarrow$

$\alpha=$ acceleration of oil droplet

$\tau_{w o}=$ modified droplet relaxation time

$d$ = diameter of droplet

$C_{D}=$ drag coefficient

$\mathrm{Re}=$ Reynolds number

$G_{k, m}=$ the generation of turbulence kinetic energy

$\mu_{t, m}=$ turbulence viscosity

$\kappa=$ turbulence kinetic energy

$\mathcal{E}=$ dissipation rate

$\mathrm{L}=$ hydraulic diameter of the inlet

$\mathrm{A}=$ inlet area

$\mathrm{S}=$ inlet circumference

$\mathrm{X}=$ insertion depth

$\mathrm{E}=$ separation efficiency

$k_{o}=$ oil concentration of the overflow

$k_{i}=$ oil concentration of the inlet

$Q_{o}=$ flow rate of the overflow

$Q_{i}=$ flow rate of the inlet

$\Delta P=$ pressure drop between inlet and underflow

$p_{i}=$ inlet pressure

$p_{u}=$ pressure of underflow

$\mathrm{d}=$ diameter of vortex

$\alpha=$ volume fraction

$\mathrm{I}=$ turbulence intensity

\section{SUBSCRIPTS}

$$
\begin{gathered}
\mathrm{m}=\text { mixture } \\
\mathrm{o}=\text { oil } \\
\mathrm{w}=\text { water }
\end{gathered}
$$

\section{REFERENCES:}

[1] Oropeza-Vazquez C, Afanador E, Gomez L, et al. OilWater Separation in a Novel Liquid-Liquid Cylindrical Cyclone (LLCC)Compact Separator-Experiments and Modeling [J]. Journals of Fluids Engineering, 2004, 126: 553-564.

[2] Listrnik J. Some Factors Influencing the Performance of De-Oiling Hydrocyclones for Marine Applications [C] England,International Conference on Hydrocyclones, September 1984, 2: 19-21.

[3] Seyda B. Separation of a Light Dispersion in a Cylindrical Vortex Chamber. Report, Michigan State University, 1991.

[4] Chu Liang-Yin, Chen Wen-Mei, Lee Xiao-Zhong. Effect of Structure Modification on Hydrocyclone Performance [J]. Separation and Purification Technology, 2000, 21: 71-86.

[5] Belaidi A, Thew M T, Munaweera S. Drop Size Effects on a De-watering Hydrocyclone. BHR Group Vortex Separation, 2000: 119-129.

[6] Rajkumar S Mathiravedu. Control system Development and Performance Evaluation of LLCC Separators [C]. Ph.D. Thesis, The University of Tulsa, 2001.

[7] Jiang Ming-hu, Zhao Li-xin, Wang Zun-ce. Effects of Geometric and Operating Parameters on Pressure drop and Oil-Water Separation Performance for Hydrocyclones [J]. The International Society of Offshore and Polar Engineers, 2002, 12: 102-106.

[8] Lucía Fernández Martínez, Antonio Gutiérrez Lavín. Manuel María Mahamud and Julio L. Bueno, Vortex finder optimum length in hydrocyclone separation [J]. 47: 192 199.

[9] Dyakowski T, Williams R A. Modelling Turbulent Flow within a Small-Diameter Hydrocyclone [J]. Chemical Engineering Science, 1993, 48(6): 1143-1152.

[10]Hirai S , Takachi T ,Matsumoto M. Predicitons of the Laminarization Phenomena in an Axially Rotating Pipe Flow [J]. J. Fluids Eng., 1988, 110: 424-430.

[11]Malhotar A, Branion R M R, Hauptmann E G. Modelling the Flow in a Hydrocyclone [J].The Canadian Journal of Chemical Engineering, 1994, 72: 953-960.

[12]He P, Salcudean M, Gartshore I S. A Numerical Simulation of Hydrocyclones [J].Trans IChemE, 1999, 77: 429-441.

[13] Grady S A, Wesson G D, Abdullah M, et al. Prediction of 10-mm Hydrocyclone Separation Efficiency Using Computational Fluid Dynamics [J]. Filtration and Separation, 2003, 40 (9): 41-46

[14]Andrew Escue, Jie Cui. Comparison of turbulence models in simulating swirling pipe flows [J]. Applied Mathematical Modelling, 2010: 1-10.

[15] 史仕荧, 吴应湘, 郑之初. 柱形旋流分离器内溢流管结 构优化分析[J]. 中国造船，2009:358-362 